\title{
HIGH FREQUENCY TWO-PLATE CAPACITIVE WIRELESS POWER TRANSFER SYSTEM
}

\author{
Kaspars Kroics, Janis Voitkans \\ Riga Technical University, Latvia \\ kaspars.kroics@gmail.com, janis.voitkans@rtu.lv
}

\begin{abstract}
The paper discusses the capacitive wireless power transfer (WPT) system for small portable device charging. The capacitive WPT can replace a conventional inductive coupling WPT, because it has no electromagnetic interference, but the capacitive WPT has limitation due to too small coupling capacitance. Therefore, to utilize the capacitive WPT system the switching frequency should be as high as possible: therefore, challenges associated with the design of the power converter working in $\mathrm{MHz}$ range will be discussed in details. A calculation of passive components, matching the network and capacitor plate design methodology is given in the paper. The design of the prototype of the capacitive WPT will be described in the paper. This paper proposes to use two copper plates to transfer power, the plates are adopted to generate electric fields and transfer power from the primary side to the secondary side. The equations for calculation of capacitance of the plates are given in the paper. An important task is to calculate the resonant network that generates high voltage in the circuit for high power transfer. The high switching of inverter transistors requires high performance transistors with optimized driver circuit. The design of the inverter and driver circuit is discussed and experimental results are shown.
\end{abstract}

Keywords: wireless power transfer, high frequency converter, capacitance, inverter.

\section{Introduction}

Nowadays, WPT systems have become more and more widely used and investigated, the review of scientific achievements in the field of WPT is given in [1-3] and in other publications. Main benefit from WPT implementation is that no wire is needed anymore to transfer energy between the power source and load. This allows to develop devices that are user friendly and easy in the exploitation. The elimination of the physical connection between the charging station and the energy consumer simplifies the charging process and removes safety concerns. The WPT system should comply with the relevant safety standards for the magnetic field. WPT technology finds its application in electrical vehicles [4-9], electronic gadgets charging [10], medical applications [11] and in many other applications. A promising WPT application is dynamic charging of electric vehicles [12] by placing WPT transceiver side under the road. Such approach allows increasing of the electric vehicle market, because in such a way the energy storage capacity requirement can be reduced and the range limit of electric vehicles extended. The WPT charging system of electric vehicles can be designed with high efficiency [13] comparable with traditional contact charging system efficiency [14-16].
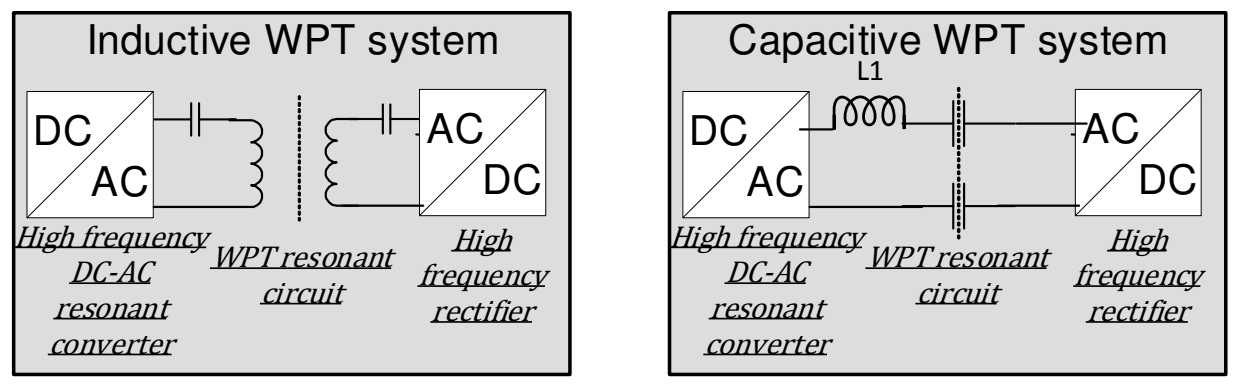

Fig. 1. Basic concepts of inductive and capacitive WPT systems

Non-radiation based WPT technologies are two - inductive power transfer that is used very widely and capacitive power transfer [17]. The basic concept of both WPT systems is shown in Fig. 1, the inductive WPT coil leakage inductance should be compensated by capacitance based resonance network and the resonance network also should also be designed for capacitive WPT. For the high frequency signal generation traditional power electronics topologies can be used and also developed more advanced new topologies [18-20]. A good comparison between both technologies is given in [21], the capacitive power transfer is more suitable for a smaller air gap and lower power in comparison with the inductive power transfer [21] although in several papers the WPT system for high 
power is reported [22;23]. The new wide band gap semiconductor devices allow increasing the switching frequency [24], therefore, capacitive power transfer can be applicable for higher power transfer and larger air gap applications in near future.

The inductive WPT system has its own drawbacks and the use of this technology is problematic in many fields. The inductive WPT system has the EMI issue, electromagnetic radiation is proven to have adverse effects on the environment and health [3] of the people exposed to the radiation, therefore shielding is required. The shielding and use of magnetics makes the cost of such system extremely high [4]. This motivates researchers to find alternative forms of wireless energy transfer.

\section{Basics of capacitive wireless power transfer system}

Capacitive wireless power transfer is based on the concept of two surfaces separated by a small air gap or a dielectric material forms capacitance, the basic equation to calculate capacitance of two parallel plates is as follows:

$$
C=\frac{\varepsilon_{r} \varepsilon_{0} S}{d},
$$

where $C$-capacitance, F;

$S$ - area of overlap of the two plates, $\mathrm{m}^{2}$;

$\varepsilon_{r}$ - relative static permittivity (sometimes called the dielectric constant) of the material between the plates (for a vacuum, $\varepsilon_{r}=1$ );

$\varepsilon_{0}$ - electric constant $\left(\varepsilon_{0} \approx 8.854 \times 10^{-12} \mathrm{~F} \cdot \mathrm{m}^{-1}\right)$;

$d$-separation between the plates, $\mathrm{m}$.

The capacitive wireless power transfer system is well proposed to transfer power through dielectric material with $\varepsilon_{r}>1$ (Fig. 2) as the capacitance value between the plates in this case is larger and power transfer is easier.

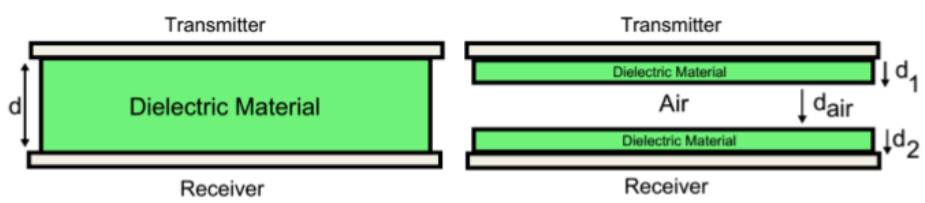

Fig. 2. Capacitive wireless charger plate possible structure [25]

According to [26] load together with the full-wave rectifier can be replaced by equivalent load $R^{\prime}{ }_{\text {load }}$ :

$$
R_{\text {load }}^{\prime}=\frac{8}{\pi^{2}} \frac{U_{2}^{2}}{P_{2}} .
$$

Eq. 2 shows that for verification of wireless power transfer, the equivalent resistive load without rectifier can be used.

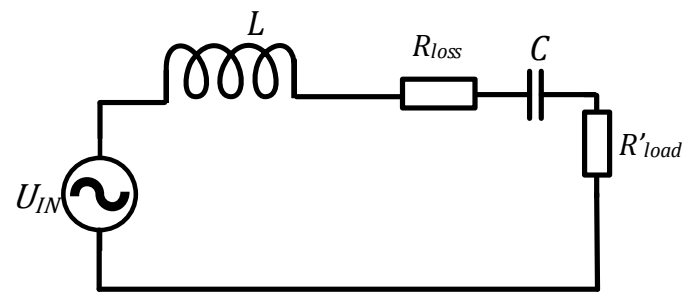

Fig. 3. Simplified equivalent circuit of capacitive WPT system

For a simplified analysis of the circuit, the input voltage can be replaced by a sinusoidal voltage source as the resonant network is tuned on fundamental frequency of the square shape voltage. If only capacitances between the plates are considered, the WPT circuit with simple series inductance compensation can be described with a simple equivalent circuit presented in Fig. 3. For such compensation circuits, the resonant inductor can be chosen to compensate all capacitance of the plates: 


$$
L=\frac{1}{\left(2 \pi f_{\text {res }}\right)^{2} C} \text {. }
$$

The voltage on wireless power plates can be calculated as follows:

$$
\dot{U}_{C}=\frac{\dot{I}_{\text {load }}}{j\left(2 \pi f_{\text {res }}\right)^{2} C} \text {. }
$$

As Eq. 4 shows, to transfer larger power to the load (larger load current), the voltage on the capacitor plates should be increased (it is limited due to safety reasons and isolation of the dielectric material) or the resonance frequency (switching frequency) should be increased. As Eq. 3 shows, by increasing the switching frequency also the value of inductance needed to compensate, the challenge associated with the increase of frequency is increased switching losses of semiconductor devices and losses in the inductor. The larger air gap between the plates means smaller capacitance and following higher voltage to transfer large power in case of a significant air gap. From this follows that capacitive WPT has limitations to transfer large power through a large air gap or plates with a large surface area are required.

\section{Design of GaN transistor based full bridge high frequency inverter}

Gallium Nitride $(\mathrm{GaN})$ based semiconductors provide advantages over conventional Silicon (Si) power devices, as shown in Fig. 4. High electron mobility of GaN transistors allows to reduce onresistance of transistors, this allows achieving high current capability with smaller die; therefore, input and output capacitances are lower than in the case of the Si transistors. Lower capacitance means that faster switching on and off is possible [27].

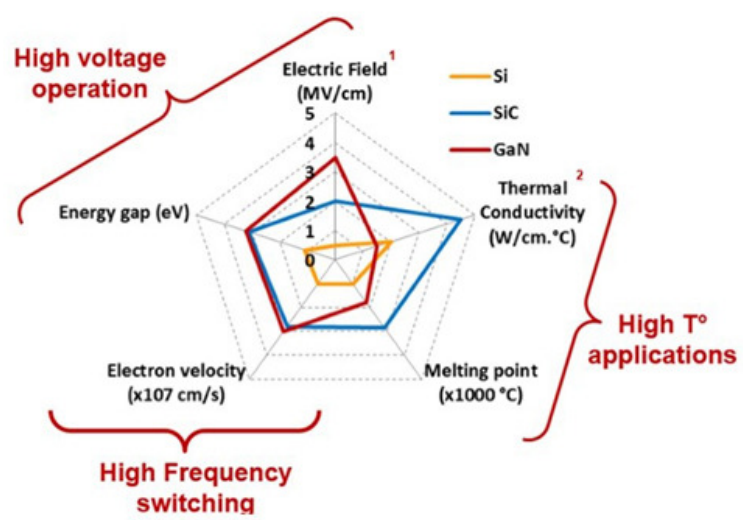

Fig. 4. Comparison of different types of transistor materials [28]

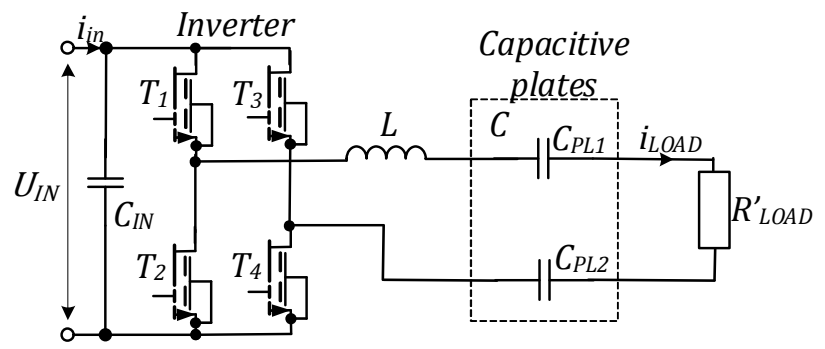

Fig. 5. GaN Transistor full bridge converter

The gate control of GaN transistors differs from typical silicon based MOSFETs. One of the main differences in terms of the gate control is the gate threshold voltage $V_{G S(t h)}$, the gate plateau and the

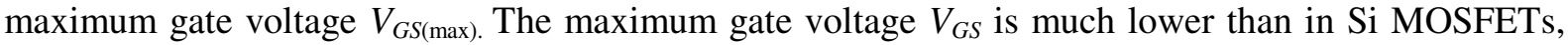
but anyway most GaN transistors are not fully turned on until $V_{G S}$ reaches about $4 \mathrm{~V}$ [29]. To turn off the GaN transistor, the gate voltage must be kept below the minimum gate threshold voltage, which is $1.3 \mathrm{~V}$ in this case. There are small number sof integrated circuits on the market that fit the requirements of GaN transistors. Silicon Labs produces isolated gate driver integrated circuits that are 
suitable for $\mathrm{GaN}$ devices, compatible with voltages in the $\mathrm{kV}$ range. The digital isolation in these drivers is based on radio frequency; therefore, the driver has high common-mode transient immunity. Although common-mode transient immunity of the driver is high, the printed circuit board should be designed very carefully to limit stray inductances and capacitances. As gate driver Si8271 integrated circuit is used, specific for GaN transistors the isolated DC-DC converter is used as power supply for both low side and high side driver circuits, because in the case of a non-isolated gate driver the gate current flows through parasitic inductance causing grounding problems.

To protect the gate from voltage spikes above the threshold or maximum rating Zener diodes should be applied between $\mathrm{G}$ and $\mathrm{S}$ terminals, a pull down resistor equal to $10 \mathrm{k} \Omega$ should be connected to prevent false turn on. To minimise parasitic gate inductance, the driver should be placed as close as possible to the transistor gate. As GaN transistors have extremely low $Q_{G}$ and drive loss, $0603 \mathrm{SMD}$ resistors can be used and the driver placed near the gate. Gate resistor allows determining the switching speed. High value of $R_{G(o n)}$ slows down switching and increases loss, whereas too small $R_{G(o n)}$ leads to high $d v / d t$ that can cause gate oscillations and additional losses [30]. High $d v / d t$ causes high common mode currents; therefore, it is important to minimise coupling capacitances of isolated power supply and use a gate driver with high common mode transient immunity. The converter circuit is shown in Fig. 5, the full bridge high frequency inverter consists of four GS66508P GaN transistors. These transistors have package with low inductance of inner connections, the device is bottom side cooled, so the thermal vias should be introduced into PCB and a heat sink placed from the opposite side of the PCB.

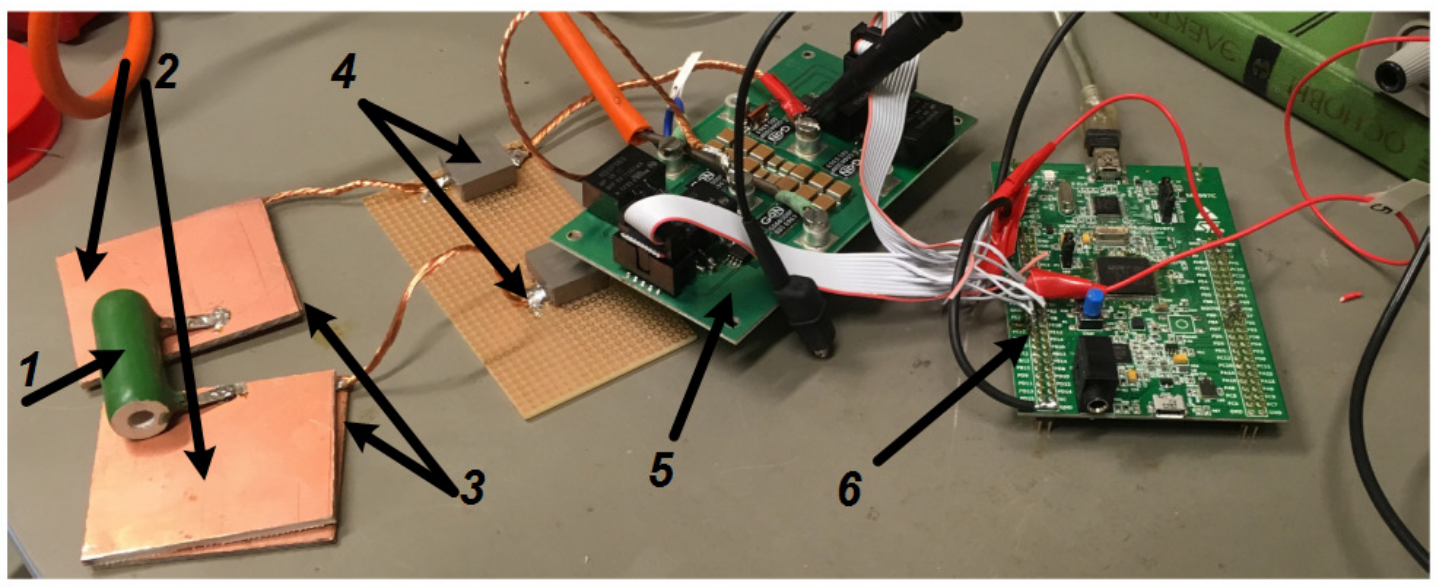

Fig. 6. Photo of experimental prototype: top side and bottom side of PCB

In order to test the capacitive WPT system the experimental prototype shown in Fig. 6 is developed. The load (1) is connected to the receiver plates (2), transceiver plates (3) are separated from receiver plates by the textolite, compensating inductances (4) are connected in series with transceiver plates, the full bridge GaN transistor based inverter with ceramic capacitors on DC bus (5) is used to generate high frequency voltage, microcontroller board (6) is used to control the transistors.

Fig. 7 shows control signals that are measured on the microcontroller output pins. As the switching frequency gets higher, the dead time is relatively larger to pulse length and this limits the maximum possible frequency. Minimum needed dead time mainly is determined by driving integrated circuit mismatch times and therefore can be concluded that for very high frequency generation a specific driver circuit is required. Also the frequency of the clock of the timer of the microcontroller determines the possibility to generate precision PWM signals and for very high frequencies, therefore, an analog pulse generator can beneficial. Nevertheless, till several $\mathrm{MHz}$ there were no problems to use digital control in combination with $\mathrm{GaN}$ transistors to generate high frequency voltage.

The measured capacitance of both plates is equal to $200 \mathrm{pF}$, to compensate this capacitance two inductors were used to work in resonance. Increasing the frequency over $1 \mathrm{MHz}$ that was expected close to the calculated resonance frequency, the resonance current have ripples that can be seen in Fig. 8. This can be explained by the fact that the inductor consists of a ferrite core that is desired for frequencies below $2 \mathrm{MHz}$ and the windings have parasitic capacitances that start to play role in case of 
high frequency. Also both plates were placed not far from each other, therefore, the cross capacitances between all plates have some influence.

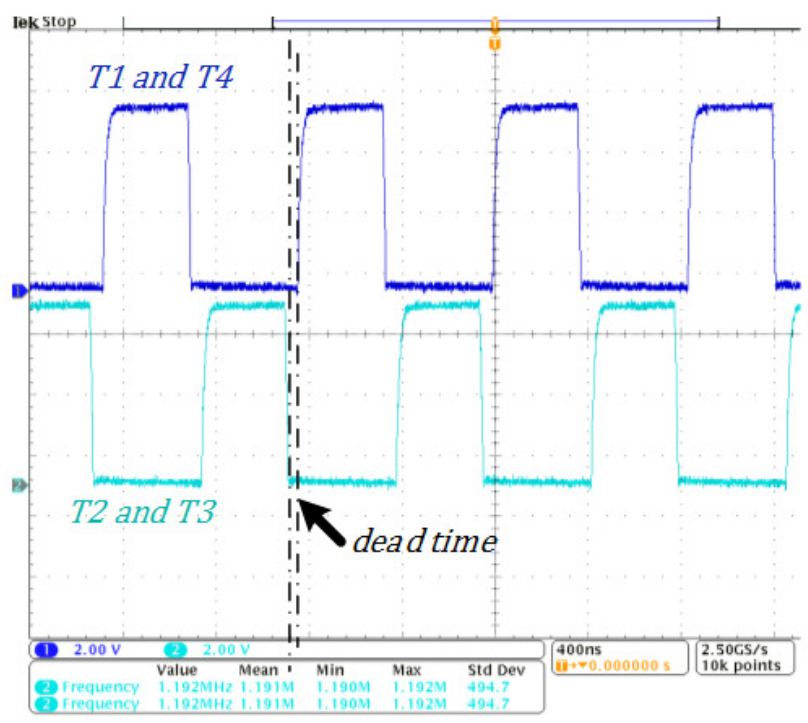

Fig. 7. Control signals of inverter

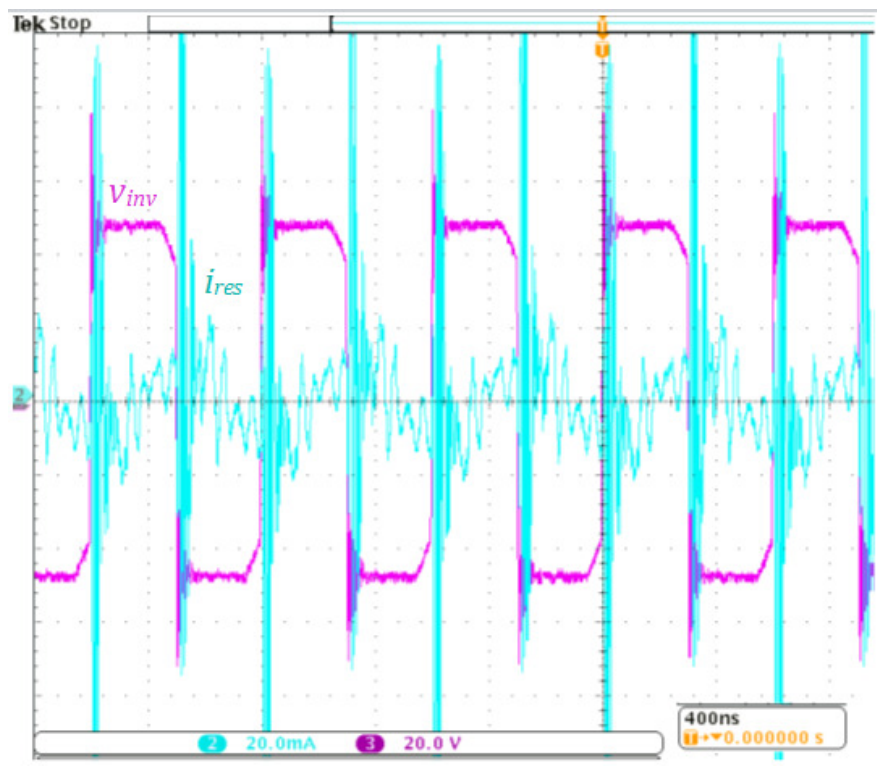

Fig. 8. Voltage on resonant tank input and resonant current

Fig. 8a shows that typical ferrite can be used till about $1 \mathrm{MHz}$ frequency, improved ferrite types till several $\mathrm{MHz}$, special ferromagnetic materials can be used till several tens of $\mathrm{MHz}$, but they have low magnetics permeability and therefore the impact on the magnetic component volume is low.

a)

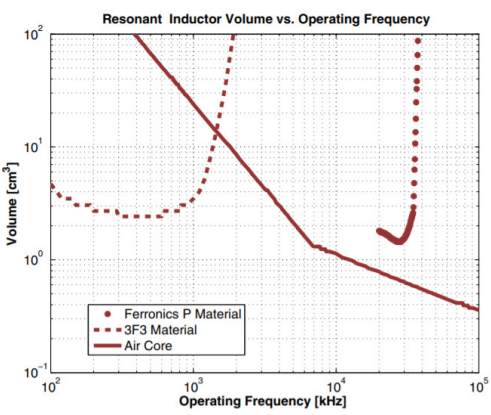

b)

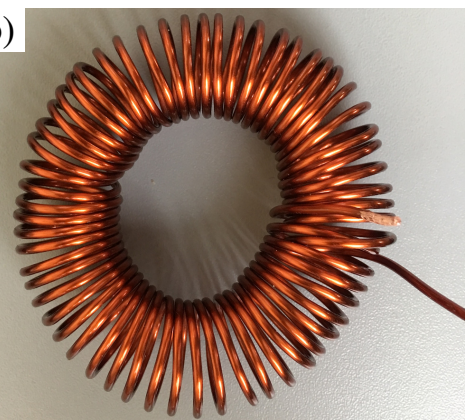

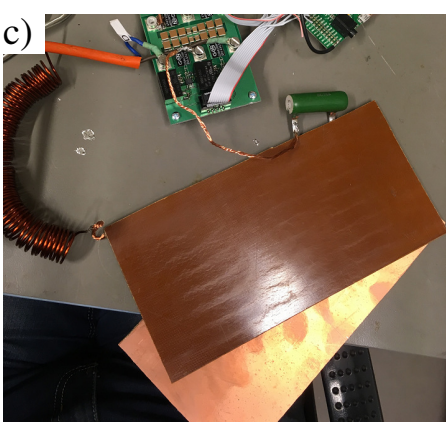

Fig. 8. Modified system: a) inductor volume dependence on frequency [31]; b) developed air core inductor; c) modified experimental capacitive WPT system 
Also for switching frequencies above $\mathrm{MHz}$ only a very high quality lit wire gives reduction of skin and proximity effects, therefore, a single strand wire with increased diameter or foil winding can be used to design a high frequency inductor.

Fig. 8b shows the developed air core inductor, in this case the diameter of the wire is oversized, as the available cupper wire was utilized. As there is no ferrite, the inductance of the air core is low and to work in resonance the surface of the plates was increased and the air gap reduced to get higher capacitance. The modified experimental setup with the air core inductor and increased surface capacitive plates is shown in Fig. 8c.

\section{Results and discussion}

The frequency of the inverter is tuned to work in resonance; in this case the switching frequency is equal to $1.66 \mathrm{MHz}$, which is close to the maximum frequency that allows to generate signals without significant oscillations with the current full bridge converter prototype. As it can been seen in Fig. 9, the inverter output voltage has oscillations, this is due to high frequency.

Although the DC bus capacitor was placed as near as possible to the transistors, anyway, the trace has some parasitic resistance, also the package of the transistor has some inductance, therefore, if the input voltage is close to the maximum allowed voltage of the transistors, additional snubber circuit should be designed to prevent breakdown of the transistors. In this case the high voltage transistors (maximum voltage $650 \mathrm{~V}$ ) are used, therefore, in this case this leads to some small additional losses, but does not destroy the transistors.

Low voltage $\mathrm{GaN}$ transistors have better package, smaller on resistance and faster switching, therefore, low voltage transistors are preferred, but as also tests with voltage above $100 \mathrm{~V}$ are intended $650 \mathrm{~V}$ transistors are almost the only choice, as on the market there are transistors with breakdown voltage lower than $100 \mathrm{~V}$ and transistors with breakdown voltage equal or more than $600 \mathrm{~V}$.

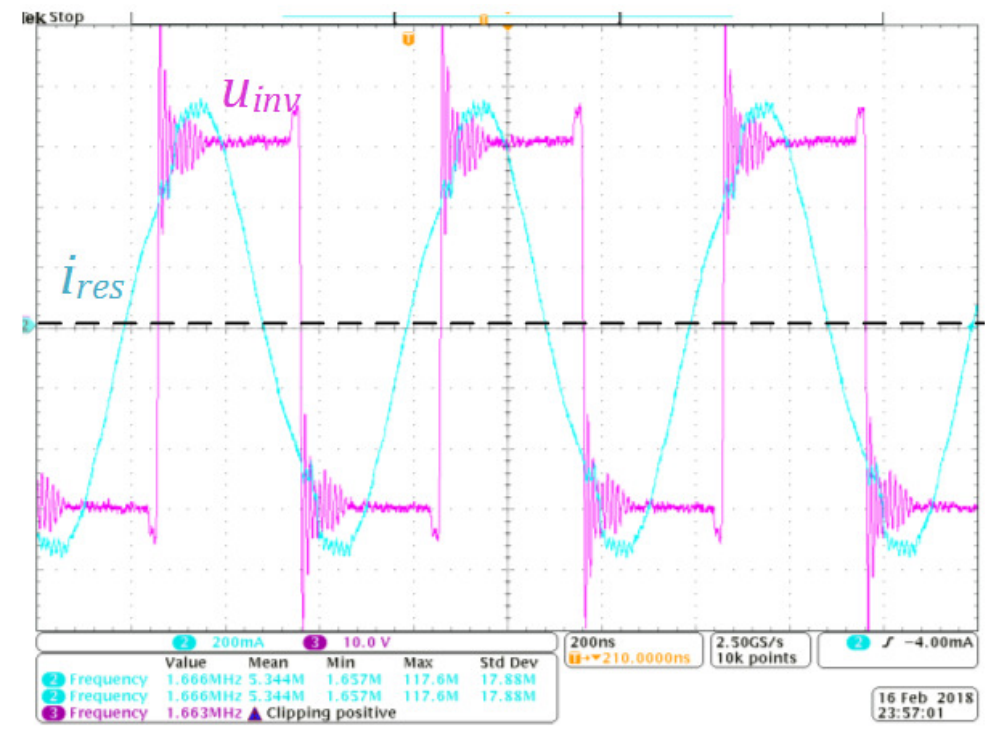

Fig. 9. Current in resonant tank $\left(i_{\text {res }}\right)$ and inverter output voltage $\left(u_{\text {inv }}\right)$

Fig. 10 shows voltage between both capacitance plates that is relatively high, as in this case the input voltage is equal to about $30 \mathrm{~V}$, the voltage between the plates reaches almost $150 \mathrm{~V}$ voltage. To transfer higher power the voltage can reach even several kilovolts. This is one of the disadvantages of the capacitive WPT system, as such high application can be dangerous or even lead to isolation electrical breakdown between the plates.

The control of the output voltage by means of frequency or pulse with modulation with the digital controller can be challenging due to low resolution at such high frequencies, therefore, an additional DC-DC converter can be used to regulate the needed power. 


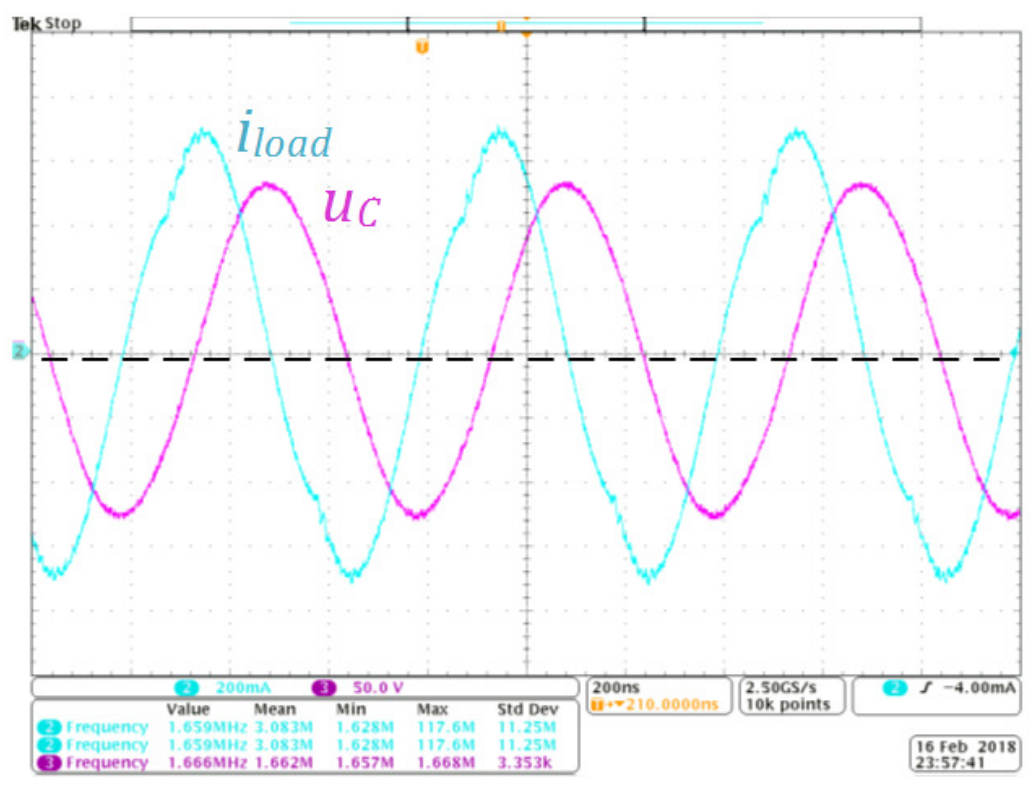

Fig. 10. Current in resonant tank and voltage between plates $\left(u_{C}\right)$

\section{Conclusions}

The capacitive WPT can be useful in some applications, especially in case, if placement of plates with a large surface is not a problem. To transfer power by means of a capacitive coupling a high frequency inverter is required to reduce the voltage between the plates and to reduce the value of the inductance needed to provide that the converter works at resonance. High frequency inverter design is very important as each parasitic inductance can lead to undesired oscillations, application of $\mathrm{GaN}$ transistors in the high frequency inverter leads to high efficiency and power density. High frequency inductor design is a challenge, therefore modifications of the resonant network can be implemented to reduce current oscillations in the inductor and to reduce the needed value of inductance. At high frequencies the air core inductor can be used that reduces cost, but increases the electromagnetic field emissions and reduction of the volume of the inductor can be challenging.

\section{Acknowledgements}

The present research has been supported by the Latvian National Research Programme "LATENERGI".

\section{References}

[1] Knecht O., KolarJ. W.Comparative evaluation of IPT resonant circuit topologies for wireless power supplies of implantable mechanical circulatory support systems. Proceedings of' Applied Power Electronics Conference and Exposition', 2017, pp. 3271-3278.

[2] Kazmierkowski M. P., MoradewiczA. J.Unplugged but Connected: Review of Contactless Energy Transfer Systems.IEEE Industrial Electronics Magazine, vol. 6, no. 4, 2012, pp. 47-55.

[3] PatilD., McDonoughM. K., MillerJ. M., FahimiB., BalsaraP. T.Wireless Power Transfer for Vehicular Applications: Overview and Challenges. IEEE Transactions on Transportation Electrification, vol. 4, no. 1, 2018, pp. 3-37.

[4] MouX., GrolingO., SunH.Energy-Efficient and Adaptive Design for Wireless Power Transfer in Electric Vehicles.IEEE Transactions on Industrial Electronics, vol. 64, no. 9, 2017, pp. 72507260.

[5] MohamedA. A. S., LashwayC. R., MohammedO., Modeling and Feasibility Analysis of QuasiDynamic WPT System for EV Applications.IEEE Transactions on Transportation Electrification, vol. 3, no. 2, 2017, pp. 343-353.

[6] Li S.,MiC. C.Wireless Power Transfer for Electric Vehicle Applications. IEEE Journal of Emerging and Selected Topics in Power Electronics, vol. 3, no. 1, 2015, pp. 4-17. 
[7] GraursI., VizulisA., RubenisA., Laizāns A.Wireless energy supply to public transport units with hybrid drive - trends and challenges. Transport and Telecommunication, vol. 15, no 1,2014, pp. 67-76.

[8] SaltanovsR., KrivchenkovA., Krainyukov A.Analysis of effective wireless communications for V2G applications and mobile objects.Proceedings of " 58 th International Scientific Conference on Power and Electrical Engineering of Riga Technical University", 2017, pp. 1-5.

[9] Saltanovs R., GalkinI.Method of adjustment and stabilization of parameters for wireless energy transfer system.Proceedings of "19th European Conference on Power Electronics and Applications" (EPE'17 ECCE Europe), 2017, p. P.1-P.6.

[10] XuW., LiangW., PengJ., LiuY., WangY.Maximizing Charging Satisfaction of Smartphone Users via Wireless Energy Transfer.IEEE Transactions on Mobile Computing, vol. 16, no. 4, 2017 pp. 990-1004.

[11] KnechtO., BosshardR., KolarJ. W.,StarckC. T.Optimization of Transcutaneous Energy Transfer coils for high power medical applications.Proceedings of "15th Workshop on Control and Modeling for Power Electronics', (COMPEL), 2014, pp. 1-10.

[12]LiuY., MaiR., LiuD., LiY., HeZ.Efficiency Optimization for Wireless Dynamic Charging System with Overlapped DD Coil Arrays.IEEE Transactions on Power Electronics, vol. PP, no. 99, 2017, pp. 1-1.

[13] Bosshard R., KolarJ. W.All-SiC 9.5 kW/dm3 On-Board Power Electronics for $50 \mathrm{~kW} / 85 \mathrm{kHz}$ Automotive IPT System.IEEE Journal of Emerging and Selected Topics in Power Electronics, vol. 5, no. 1, 2017, pp. 419-431.

[14] PanfilovD., HusevO., BlaabjergF., ZakisJ.,KhandakjiK.Comparison of three-phase three-level voltage source inverter with intermediate $\mathrm{dc}$-dc boost converter and quasi-Z-source inverter.IET Power Electronics, vol. 9, no. 6, 2016, pp. 1238-1248.

[15] VinnikovD., ZakisJ., HusevO., StrzeleckiR.New high-gain step-up DC/DC converter with highfrequency isolation.Proceedings of' Twenty-Seventh Annual IEEE Applied Power Electronics Conference and Exposition"' (APEC), 2012, pp. 1204-1209.

[16]KroicsK., SirmelisU., GrigansL., BrazisV.Digitally controlled 4-phase interleaved DC-DC converter with coupled inductors for storage application in microgrid.Proceedings of "9th International Conference on Compatibility and Power Electronics'”(CPE), 2015, pp. 504-509.

[17]LiS., LiuZ., ZhaoH., ZhuL., ShuaiC., ChenZ.Wireless Power Transfer by Electric Field Resonance and its Application in Dynamic Charging.IEEE Transactions on Industrial Electronics, vol. 63, no. 10, 2016, pp. 6602-6612.

[18]ZakisJ., VinnikovD., BisenieksL.Some design considerations for coupled inductors for integrated buck-boost converters.Proceedings of "International Conference on Power Engineering, Energy and Electrical Drives", 2011, pp. 1-6.

[19] SirbuI. G., MandacheL.Comparative analysis of different topologies for wireless power transfer systems.Proceedings of' 'International Conference on Environment and Electrical Engineering and 2017 IEEE Industrial and Commercial Power Systems Europe', 2017, pp. 1-6.

[20]LiivikL., ChubA., VinnikovD.,ZakisJ.Experimental study of high step-up quasi-Z-source DC-DC converter with synchronous rectification.Proceedings of "International Conference on Compatibility and Power Electronics" (CPE), 2015, pp. 409-414.

[21] Dai J., LudoisD. C.A Survey of Wireless Power Transfer and a Critical Comparison of Inductive and Capacitive Coupling for Small Gap Applications.IEEE Transactions on Power Electronics, vol. 30, no. 11, 2015, pp. 6017-6029.

[22]LuF., ZhangH., MiC.A Two-Plate Capacitive Wireless Power Transfer System for Electric Vehicle Charging Applications.IEEE Transactions on Power Electronics, vol. 33, no. 2, 2018, pp. 964-969.

[23] RegensburgerB., SinhaS., KumarA., VanceJ., PopovicZ., AfridiK. K.Kilowatt-scale large air-gap multi-modular capacitive wireless power transfer system for electric vehicle charging.Proceedings of "Applied Power Electronics Conference and Exposition”' (APEC), 2018, pp. 666-671.

[24] KroicsK.,ZakisJ.Electronic ballast for gas discharge lamp based on input - series output - series resonant converter.Proceedings of "International Exhibition and Conference for Power Electronics, Intelligent Motion, Renewable Energy and Energy Management'(PCIM Europe) 2017,pp. 1186-1192. 
[25] RozarioD.,AzeezN. A, WilliamsonS. S.A modified resonant converter for wireless capacitive power transfer systems used in battery charging applications.Proceedings of "IEEE Transportation Electrification Conference and Expo"' (ITEC), 2016, pp. 1-6.

[26] SteigerwaldR.L.A comparison of half-bridge resonant converter topologies.IEEE Transactions on Power Electronics, vol. 3, no. 2, 1988, pp. 174-182.

[27] KroicsK., ZakisJ., SuzdalenkoA., HusevO.Design Considerations for Gan-Based Microinverter for Energy Storage Integration Into Ac Grid.Latvian Journal of Physics and Technical Sciences, vol. 54, no. 5, pp. 14-25, 2017.

[28] "Rise of the 3rd Generation Semiconductor Silicon Carbide Technology". [online][ 04.08.2017]. Available at: http://www.iabrasive.com/articles/rise-of-the-3rd-generation-semiconductor-siliconcarbide-technology

[29] RoschattP. M., McMahonR. A., PickeringS.Investigation of dead-time behaviour in GaN DC-DC buck converter with a negative gate voltage.Proceedings of' 9 th International Conference on Power Electronics and ECCE Asia' (ICPE-ECCE Asia), 2015, pp. 1047-1052.

[30] KroicsK.Bi-directional two level 6-phase DC-DC converter for energy storage application.Proceedings of "International Exhibition and Conference for Power Electronics, Intelligent Motion, Renewable Energy and Energy Management'” (PCIM Europe), 2015, pp. 1-8.

[31] PerreaultD. J., et al.Opportunities and Challenges in Very High Frequency Power Conversion.Proceedings of' 24th Annual IEEE Applied Power Electronics Conference and Exposition', 2009, pp. 1-14. 